\title{
Structural and thermal properties of silicon-doped fullerenes
}

\author{
P. A. Marcos \\ Departamento de Física, Universidad de Burgos, E09001 Burgos, Spain \\ J. A. Alonso \\ Departamento de Física Teórica, Universidad de Valladolid, E-47011 Valladolid, Spain \\ L. M. Molina \\ Institute of Physics and Astronomy, University of Aarhus, DK-8000 Aarhus C, Denmark \\ A. Rubio \\ Departamento de Física de Materiales, Universidad del País Vasco and DIPC, Apdo. 1072, \\ 20018 San Sebastián, Spain \\ M. J. López \\ Departamento de Física Teórica, Universidad de Valladolid, E-47011 Valladolid, Spain
}

(Received 12 February 2002; accepted 25 February 2003)

\begin{abstract}
Extensive Molecular Dynamics simulations have been performed to investigate the structural and thermal properties of Si-doped fullerenes containing one and two silicon atoms. Both, a many-body potential and $a b$ initio Density Functional Theory (DFT) have been used to investigate the structural features of the heterofullerenes. The competition between the exohedral and the substitutional types of doping, as a function of fullerene size (both even and odd heterofullerenes have been considered) and Si concentration, is analyzed. The DFT calculations confirm the main structural trends obtained with the many-body potential. The thermal stability and the structural transformations of the heterofullerenes have been also studied as a function of temperature $(T=0-5000 \mathrm{~K})$. The structural transformations include, local rearrangement of atoms, isomerization transitions, diffusion of atoms, eventual destruction of the cage, and sublimation of atoms. The isomerization transition between exohedral and substitutional isomers has been observed experimentally. (C) 2003 American Institute of Physics. [DOI: 10.1063/1.1568085]
\end{abstract}

\section{INTRODUCTION}

As it is well known and exploited in many technological applications, doping is a very efficient way of modifying and tailoring the electronic, optical, conducting, etc. properties of many materials. Just to mention one example, the microelectronics, as we know it today, emerges from the use of doped semiconductors. It was not by accident, that the discovery of $\mathrm{C}_{60}$ (Ref. 1) and its production in macroscopic quantities ${ }^{2}$ prompted a number of investigations on the functionalization and doping of fullerenes. Heteroatoms have been successfully introduced (endohedral doping) in the hollow cavity of fullerenes. ${ }^{3}$ It has also been possible to attach heteroatoms, e.g., alkalis, alkaline earth metals, and transition metals, to the external surface of fullerenes (exohedral doping) both in the gas phase ${ }^{4}$ and in the solid phase. ${ }^{5}$ Another possibility is the substitution of a number of $\mathrm{C}$ atoms by heteroatoms (substitutional doping) such as boron and nitrogen. ${ }^{6-8}$

Silicon is a very interesting element for doping fullerenes. On the one hand, $\mathrm{SiC}$ compounds and alloys exhibit outstanding properties such as polymorphism, extraordinary hardness, variable band gaps, etc. On the other hand, the hollow-cage structure of fullerenes offers a unique framework for developing new $\mathrm{SiC}$ compounds. Although silicon is isoelectronic with carbon it exhibits quite different physical and chemical properties, e.g., it does not develop $s p^{2}$ networks. On the contrary it prefers three-dimensional struc- tures. Moreover, $\mathrm{Si}-\mathrm{C}$ bonds are typically $30 \%$ longer than $\mathrm{C}-\mathrm{C}$ bonds. These features would play against the substitutional incorporation of silicon in fullerenes. However, Sidoped fullerenes have been synthesized ${ }^{9-13}$ in recent years by several groups both in substitutional and exohedral configurations. Silicon-doped fullerenes where first produced by laser ablation of $\mathrm{Si} / \mathrm{C}$ composite rods. ${ }^{9}$ The direct growth of heterofullerenes favors the substitutional incorporation of silicon in the fullerene network. ${ }^{9,11}$ Photofragmentation experiments ${ }^{14}$ have shown heterofullerenes containing up to seven substitutional silicon atoms. Si-doped fullerenes can also be produced by mixing a vapor of preformed $\mathrm{C}_{60}$ fullerenes with a silicon vapor in an inert gas condensation cell. ${ }^{12}$ This technique produces exohedrally doped fullerenes. However, under laser irradiation the exohedrally doped fullerenes transform into substitutionally doped fullerenes. ${ }^{12}$ Moreover, the coverage of $\mathrm{C}_{60}$ with silicon is very limited. In contrast to metals, silicon does not wet the fullerene surface and prefers clustering. ${ }^{13}$ It is, then, of interest to understand the interplay between the substitutional and the exohedral types of doping, the possible transformations between these two structures and their relationship with the production techniques.

$A b$ initio calculations have been performed for studying the structural and electronic configuration of "substitutional" Si-doped $\mathrm{C}_{60}$ fullerenes containing one and two $\mathrm{Si}$ 
atoms. $^{11,12,15}$ The HOMO-LUMO gap decreases as the number of silicon atoms in the fullerene cage is increased and the corresponding Kohn-Sham orbitals are mainly localized on the silicon atom. These results indicate that the silicon atom may act as a "distinct" reactive site in fullerenes promoting their self-assembly. Recent $a b$ initio calculations ${ }^{16}$ indicate that is possible to substitute up to 12 silicon atoms in $\mathrm{C}_{60}$ without distorting the original structure too much. On the other hand, the thermal stability of pure fullerenes has been studied experimentally both for fullerenes in the gas phase ${ }^{17}$ and in the condensed phase (fullerite). ${ }^{18}$ However, for Sidoped fullerenes, this property remains largely unexplored. It is our intention to contribute to the understanding of the thermal stability of doped fullerenes.

In this paper we study, for the first time, the competition between the substitutional and the exohedral types of $\mathrm{Si}$ doping in fullerenes, and the thermal behavior of these heterofullerenes. Our investigation will help to disclose the interplay between the size and concentration of the heterofullerenes and their preferential type of doping, substitutional or exohedral. Moreover, our results give insight on the heterofullerenes synthesized by different techniques. Both even and odd heterofullerenes containing one and two silicon atoms are considered $\left(\mathrm{C}_{n} \mathrm{Si}, n=58-61\right.$ and $\mathrm{C}_{n} \mathrm{Si}_{2}$, $n=58,60,78$ ). The realistic (empirical) Tersoff potential ${ }^{19,20}$ is used for mimicking the interactions in the heterofullerenes. We have also performed ab initio calculations, using Density Functional Theory (DFT) techniques, for some selected heterofullerenes. The DFT results confirm the main structural features obtained with the empirical potential. This gives us confidence to use the potential in extensive Molecular Dynamics simulations for investigating the thermal stability, the dynamical characteristics, and the structural transformations of the heterofullerenes as a function of temperature ( $T$ $=0-5000 \mathrm{~K}$ ).

The structure of the paper is as follows: Section II gives a brief description of the model (Tersoff) potential and the $a b$ initio (DFT) techniques used in the calculations. It also describes the Molecular Dynamics simulations performed here. In Sec. III we present the simulation results. First the structural features of Si-doped fullerenes and then their thermal behavior. A brief summary follows in Sec. IV.

\section{THEORETICAL BACKGROUND}

An extensive search of isomers as a function of cluster size and composition (for one and two silicon atoms per fullerene) and extensive Molecular Dynamics simulations have been performed using the realistic, but necessarily approximate, Tersoff's potential. ${ }^{19,20}$ This potential, which mimics covalent interatomic interactions, is based in the bond order ideas introduced by Abell, ${ }^{21}$ i.e., the strength of a bond depends on the local environment. The bond order concept led to a many-body potential depending on coordination and on the bond angles. The potential is flexible enough for describing both the $s p^{2}$ and $s p^{3}$ hybridizations of carbon. Formally the potential energy is written as a sum of pairwise interactions,

$$
V=\frac{1}{2} \sum_{i, j(\neq i)=1}^{N} f_{\mathrm{c}}\left(r_{i j}\right)\left[f_{\mathrm{R}}\left(r_{i j}\right)+b_{i j} f_{\mathrm{A}}\left(r_{i j}\right)\right],
$$

where $N$ is the number of atoms in the system, $f_{R}$ and $f_{A}$ are the repulsive and attractive pair potential functions, respectively, given by

$$
\begin{aligned}
& f_{\mathrm{R}}\left(r_{i j}\right)=A_{i j} e^{\left(-\lambda_{i j} r_{i j}\right)}, \\
& f_{\mathrm{A}}\left(r_{i j}\right)=-B_{i j} e^{\left(-\mu_{i j} r_{i j}\right)},
\end{aligned}
$$

and $b_{i j}$ is the bond order parameter which depends on the local environment and gives the many-body character to the potential,

$$
\begin{aligned}
& b_{i j}=\chi_{i j}\left(1+\beta_{i}^{n_{i}} \xi_{i j}^{n_{i}}\right)-\left(1 / 2 n_{i}\right), \\
& \xi_{i j}=\sum_{k(\neq i, j)=1}^{N} f_{\mathrm{c}}\left(r_{i k}\right) \omega_{i k} g\left(\theta_{i j k}\right), \\
& g\left(\theta_{i j k}\right)=1+\frac{c_{i}^{2}}{d_{i}^{2}}-\frac{c_{i}^{2}}{d_{i}^{2}+\left(h_{i}-\cos \theta_{i j k}\right)^{2}},
\end{aligned}
$$

where $\theta_{i j k}$ is the bond angle between the $i j$ and the $i k$ bonds. The free parameters of the potential $A, B, \lambda, \mu, \beta, n, c, d$, $h, \chi$, and $\omega$ are empirically adjusted. ${ }^{20} \chi$ weighs the strength of the homopolar and heteropolar bonds and $\omega$ weights the contribution of environmental atoms of different types. $f_{\mathrm{c}}$ in Eqs. (1) and (3) is a smooth cut-off function which limits the range of the potential to the close neighborhood of each atom, i.e., it damps the interatomic interactions smoothly down to zero between the first and the second neighboring shells. We have used a Fermi-type cutoff function, ${ }^{22}$

$$
f_{\mathrm{c}}\left(r_{i j}\right)=\frac{1}{1+e^{\left(r_{i j}-r_{o_{i j}}\right) / D}},
$$

which it is derivable with continuity to all orders and therefore guarantees a good conservation of energy in dynamical studies.

The study begins with the generation of a large number of substitutional and exohedral configurations of the heterofullerenes. All the structures are then relaxed to the closest local minima using the thermal quenching procedure. The annealing at high temperatures of some selected isomers will eventually produce new isomers completing the isomeric chart of the heterofullerenes. The chart of isomers gives insight on the energetic and structural features of the doped fullerenes. The finite temperature behavior of the heterofullerenes is then investigated by constant energy Molecular Dynamics simulations. The fullerenes are gradually heated up, by scaling up the velocities of all the atoms, from $0 \mathrm{~K}$ up to about $4500 \mathrm{~K}$. At each temperature, the time evolution of the system, for times between $50-125 \mathrm{ps}$, is obtained by numerical integration of Newton's equations of motion using the velocity version of Verlet's algorithm. ${ }^{23}$ A time step of 0.2 fs guarantees that, after performing 250000 integration steps (i.e., $50 \mathrm{ps}$ ), the total energy is conserved within $0.01 \%$.

The Tersoff potential has proved reliable in describing the properties of a wide variety of carbon systems ${ }^{22,24}$ as well as the properties of $\mathrm{SiC}$ and its defects. ${ }^{20}$ The extent of the simulations presented here, which include the generation of a 
large number of isomers and lengthy MD simulations (lasting in total about $1000-3000$ ps per isomer considered), make the use of more accurate quantum techniques impractical. However, to assess the actual reliability of the potential for Si-doped fullerenes we will compare the results of the Tersoff potential with ab initio DFT calculations for some selected heterofullerenes as well as with the available experiments.

The ab initio DFT simulations were performed using the "Dacapo" code ${ }^{25}$ with ultrasoft pseudopotentials, ${ }^{26}$ a plane wave basis set $\left(E_{\text {cut }}=18 \mathrm{Ryd}\right)$ and the GGA approximation (PW91 functional ${ }^{27}$ ) for the description of exchangecorrelation effects. A fcc unit cell with a very large lattice parameter $(21 \AA)$ was employed in order to make clustercluster interaction in neighboring cells negligible. Each structure was relaxed until a $0.01 \mathrm{eV}$ convergency criterion was fulfilled.

\section{RESULTS}

\section{A. Geometrical structure of one-Si heterofullerenes}

The Tersoff potential is used to perform an extensive search of possible exohedral and substitutional isomers of one $\mathrm{Si}$ doped fullerene. Then a selection of those isomers is fully optimized using $a b$ initio (DFT) techniques. First we mimic the production of exohedral Si-doped fullerenes, through the mixing of $\mathrm{Si}$ and $\mathrm{C}_{60}$ vapors in a condensation cell, ${ }^{12}$ by the soft-landing of $\mathrm{Si}$ atoms onto the external surface of $\mathrm{C}_{60}$. The $\mathrm{Si}$ atoms approach the fullerenes in quasistatic conditions (i.e., with zero velocity), following different radial directions. Silicon binds preferentially on top of a $\mathrm{C}$ atom (on-top configuration) with a binding energy of $2.3 \mathrm{eV}$ with respect to the separated $\mathrm{Si}$ and $\mathrm{C}_{60}$ subsystems. Silicon can be also attached above the midpoint of $\mathrm{C}-\mathrm{C}$ bonds ${ }^{28}$ (edge configurations) with binding energies of 1.2 and 0.6 $\mathrm{eV}$, for edges between a hexagon and a pentagon and edges between two hexagons, respectively. The fullerene cage structure remains intact upon the soft-landing of $\mathrm{Si}$ atoms. The soft-landing procedure generates exohedral isomers exclusively, in agreement with the experiments. ${ }^{12}$ However, the annealing of the exohedral heterofullerenes at high temperatures produces substitutional isomers as well (see below).

All possible exohedral and substitutional configurations of $\mathrm{C}_{n} \mathrm{Si}(n=58-61)$ have been investigated. The exohedral on-top and edge configurations are generated by placing one $\mathrm{Si}$ atom on top of every nonequivalent $\mathrm{C}$ atom and above every nonequivalent $\mathrm{C}-\mathrm{C}$ bond, respectively, of the corresponding $\mathrm{C}_{n}$ fullerenes. Similarly, starting from $\mathrm{C}_{n+1}$, the substitutional configurations of $\mathrm{C}_{n} \mathrm{Si}$ are generated by substituting every nonequivalent $\mathrm{C}$ atom in the fullerene by a $\mathrm{Si}$ atom. The well-known structures of the even $\mathrm{C}_{n}, n=58,60$, and 62 , fullerenes ${ }^{29}$ are taken as given and the structures of the odd $\mathrm{C}_{n}, n=59$ and 61 , fullerenes are generated from $\mathrm{C}_{60}$ and $\mathrm{C}_{62}$, respectively, by substituting a $\mathrm{C}_{2}$ dimer between two adjacent rings by a single $\mathrm{C}$ atom. The two rings reduce by one their respective number of edges and the $\mathrm{C}$ atom becomes fourfold coordinated. The thermal quenching procedure has been used to relax the heterofullerene structures to the corresponding closest local minima. Exohedral attach-

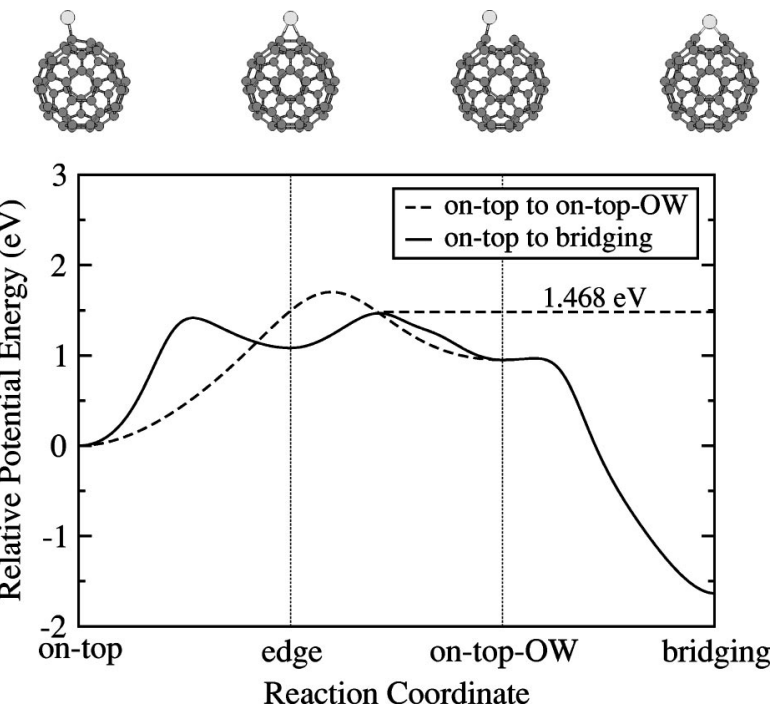

FIG. 1. Low energy paths for the transformation of the on-top isomer of $\mathrm{C}_{60} \mathrm{Si}$ into the bridge isomer. The solid line shows the pathway going from the on-top isomer to the edge configuration, then to the on-top-OW structure, and finally to the bridge isomer. The dashed line corresponds to the direct transformation of the on-top isomer into the on-top-OW structure. The reaction coordinate is defined as the average of the coordinates of two consecutive isomers in the defined pathway.

ment of Si atoms does not deform the fullerene cage whereas substitutional doping produces local distortions of the fullerenes around the $\mathrm{Si}$ atoms. The $\mathrm{Si}$ atom is pushed outwards the cage due to the larger (about 20\%) C-Si bond length. Moreover, the lowest energy substitutional isomers of odd fullerenes are obtained when the $\mathrm{Si}$ atom replaces the fourfold coordinated C atom (see Fig. 2). This could be expected from the silicon tendency to be fourfold coordinated.

To further explore possible isomers of $\mathrm{C}_{n} \mathrm{Si}$ heterofullerenes we have annealed at high temperatures some selected isomers. For instance the on-top isomer of $\mathrm{C}_{60} \mathrm{Si}$ remains stable when annealed up to temperatures of about $1900 \mathrm{~K}$ for 125 ps. However, above this temperature it becomes unstable and anneals to a lower energy configuration (we will refer to it as bridge) in which the $\mathrm{Si}$ atom binds to a "broken" $\mathrm{C}-\mathrm{C}$ edge between a hexagon and a pentagon. We say that a $\mathrm{C}-\mathrm{C}$ edge is "broken" when the two $\mathrm{C}$ atoms separate one from another while lifting slightly out from the fullerene surface producing a hole (or open window, $\mathrm{OW}$ ) in the structure of the fullerene. Broken $\mathrm{C}-\mathrm{C}$ bonds have been observed in simulations ${ }^{30}$ of all carbon fullerenes at intermediate temperatures $(T \sim 2500 \mathrm{~K})$. We have found bridge structures in all the heterofullerenes investigated, having lower energy than the corresponding edge and on-top configurations. Notice, however, that the bridge configuration should be viewed as a slight distortion of the edge structure. Figure 1 shows two low energy paths connecting the on-top and the bridge isomers of $\mathrm{C}_{60} \mathrm{Si}$. The first path runs from the on-top to the edge configuration, then back to an on-top configuration in which the $\mathrm{Si}$ atom is over a $\mathrm{C}$ atom of a broken $\mathrm{C}-\mathrm{C}$ bond (on-top-OW) and finally ends in the bridge structure. The second path connects the on-top and the ontop-OW structures through the direct break up of the corresponding $\mathrm{C}-\mathrm{C}$ bond. We find an upper bound of $1.5 \mathrm{eV}$ for 
A
C58Si 1

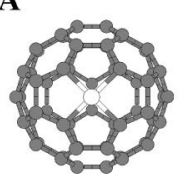

0.1744

D

$\mathrm{C}_{59} \mathrm{Si} 1$

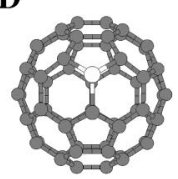

0.0

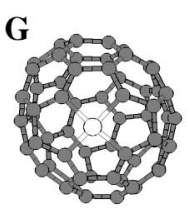

0.7562

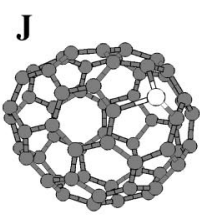

0.0

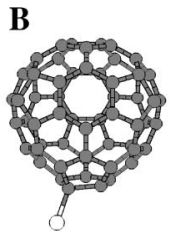

2.3126

E

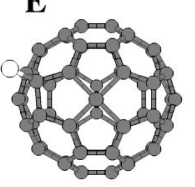

6.6421

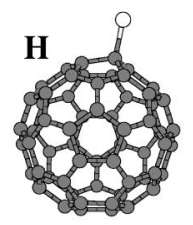

1.6339

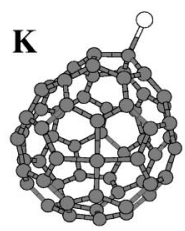

7.4265

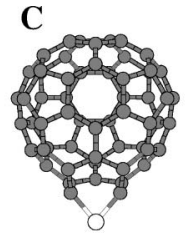

0.0

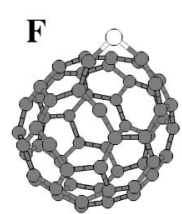

3.4829

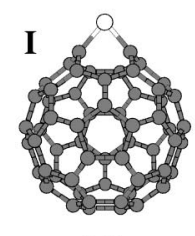

0.0

$\mathbf{L}$

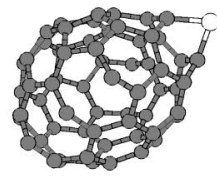

2.7249
FIG. 2. Lowest energy substitutional (first column), on-top (second column), and bridge (third column) isomers of one-Si doped fullerenes containing from 58 to 61 carbon atoms. The isomer energies with respect to the ground state are given in $\mathrm{eV}$.

the energy barrier between the on-top and the bridge isomers.

Figure 2 shows the lowest energy substitutional, on-top, and bridge isomers of $\mathrm{C}_{n} \mathrm{Si}$ heterofullerenes. The bridge structure is always lower in energy than the corresponding on-top structure. The addition and substitution reactions,

$$
\begin{aligned}
& \mathrm{C}_{n}+\mathrm{Si}+\Delta E^{\text {add }} \rightarrow \mathrm{C}_{n} \mathrm{Si}(\text { exohedral }), \\
& \mathrm{C}_{n+1}+\mathrm{Si}+\Delta E^{\text {subst }} \rightarrow \mathrm{C}_{n} \mathrm{Si}(\text { substitutional })+\mathrm{C},
\end{aligned}
$$

respectively, give insight on the competition between exohedral and substitutional isomers. Exohedral attachment of $\mathrm{Si}$ atoms to preformed $\mathrm{C}_{n}$ fullerenes $(n=58-61)$ is an exothermic process. On the other hand, the substitution reaction is exothermic in the case of odd fullerenes but it requires the supply of energy in the case of even fullerenes. These results agree with the production of exohedrally doped fullerenes upon mixing the vapors of preformed $\mathrm{C}_{60}$ fullerenes and silicon. ${ }^{12}$ Our results also justify the appearance of substitutional heterofullerenes only after some extra energy has been supplied to the sample, e.g., by laser irradiation. ${ }^{12}$ The minimum energy structures of the heterofullerenes, however, are not determined by the energetics of the addition and substitution reactions. As a general trend, we have found that the minimum energy structures are mainly determined by the tendency to reconstruct almost perfect even fullerenes. In
A

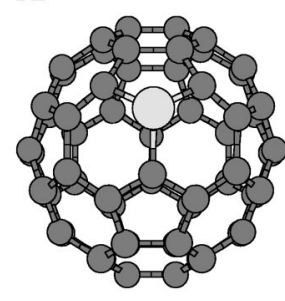

$\mathbf{0 . 0}$

C

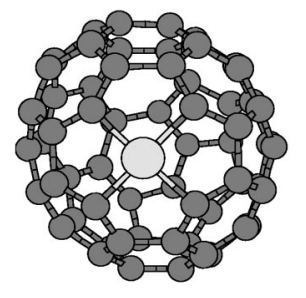

4.1
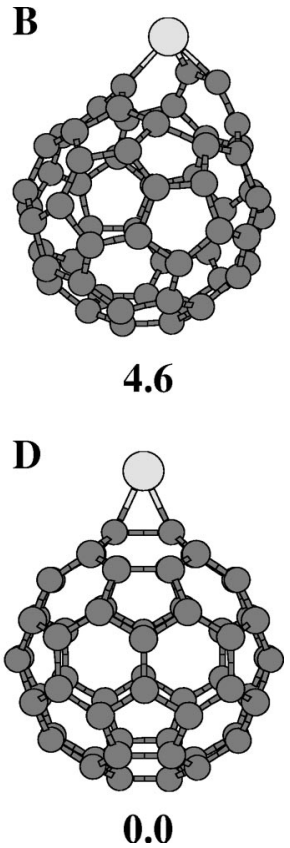

FIG. 3. Lowest energy substitutional (first column) and exohedral (second column) isomers of $\mathrm{C}_{n} \mathrm{Si}(n=59-60)$ obtained by ab initio (DFT) calculations. The isomer energies with respect to the ground state are given in $\mathrm{eV}$.

other words, the undoped underlying structure of the ground state isomer is an even fullerene. Thus, the substitutional isomer is the minimum energy structure of even heterofullerenes whereas the ground state of odd heterofullerenes corresponds to the exohedral (bridge) isomer. Our theoretical predictions are in perfect agreement with mobility experiments ${ }^{10}$ of Si-doped fullerenes which provide strong evidence of substitutional $\mathrm{Si}$ in even heterofullerenes and suggest exohedral doping in odd heterofullerenes.

DFT calculations confirm the structural and energetic trends of one Si heterofullerenes predicted by Tersoff's potential. Figure 3 shows the lowest energy substitutional and exohedral isomers of $\mathrm{C}_{59} \mathrm{Si}$ and $\mathrm{C}_{60} \mathrm{Si}$ obtained within the DFT framework. The substitutional isomer is the minimum energy structure of $\mathrm{C}_{59} \mathrm{Si}$, an even heterofullerene, whereas the ground state of $\mathrm{C}_{60} \mathrm{Si}$, an odd heterofullerene, corresponds to the exohedral-edge configuration of Si. Notice, that the exohedral-bridge structure obtained with Tersoff potential and the edge structure obtained with DFT are topologically very similar. Moreover, our DFT calculations show that the exohedral attachment of one $\mathrm{Si}$ atom on $\mathrm{C}_{60}$ is an exothermic process $\left[\Delta E^{\mathrm{add}}=-1.7 \mathrm{eV}\right.$, see Eq. (5)], whereas the substitution reaction for $\mathrm{C}_{60}$ is endothermic $\left[\Delta E^{\text {subst }}\right.$ $=5.4 \mathrm{eV}$, see Eq. (6)], also in agreement with the predictions of the Tersoff potential. Both exohedral and substitutional doping of $\mathrm{C}_{60}$ with one $\mathrm{Si}$ atom reduces its binding energy in about $0.1 \mathrm{eV} /$ atom.

\section{B. Geometrical structure of two-Si heterofullerenes}

To gain some insight on the first stages of multiple doping in fullerenes, we have also studied even heterofullerenes containing two silicon atoms, $\mathrm{C}_{n} \mathrm{Si}_{2}(n=58,60,78)$. The number of possible structural configurations of the hetero- 
A

$\mathrm{C}_{58 \mathrm{Si}} 2$
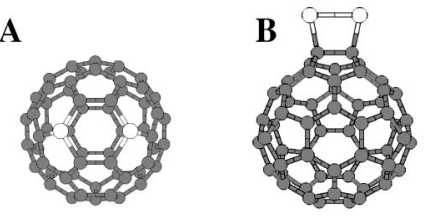

C

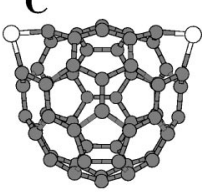

D

$\mathrm{C}_{60} \mathrm{Si}_{2}$
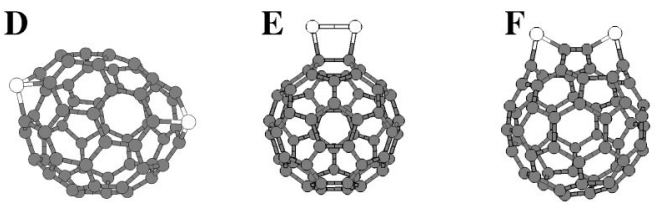

$\mathrm{C}_{78} \mathrm{Si}_{2}$

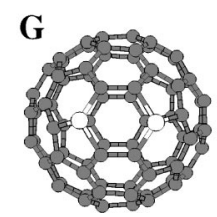

A

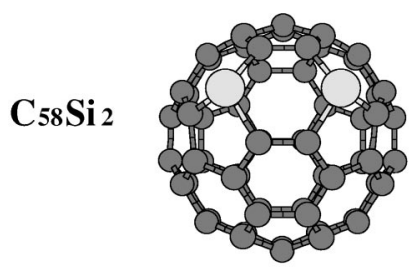

$\mathbf{0 . 0}$

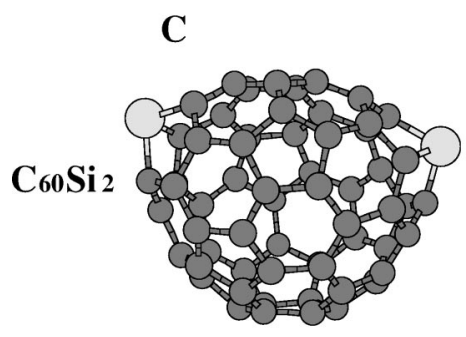

2.6

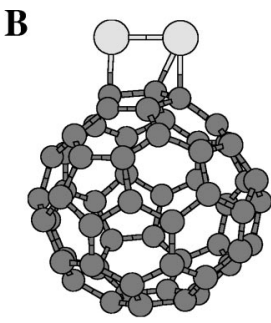

1.8

D

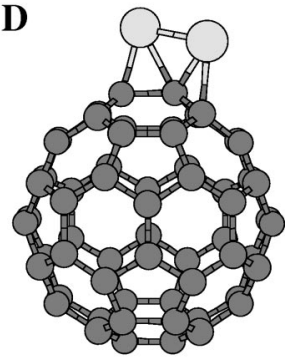

0.0

FIG. 4. Lowest energy substitutional (first column), on-top (second column), and bridge (third column) isomers of two-Si doped fullerenes containing 58, 60, and 78 carbon atoms.

fullerenes increases tremendously in going from one to two silicon atoms. Therefore, based on the structural trends observed in one Si doped fullerenes, we will restrict the search of isomers to those configurations whose undoped underlying structures are even fullerenes. Thus, we have generated all possible configurations in which both $\mathrm{Si}$ atoms are in either substitutional or exohedral, on-top or edge, configurations. Similarly to the case of one-Si fullerenes, the annealing of the on-top isomer to high temperatures yields to a lower energy structure in which both silicon atoms bind to broken $\mathrm{C}-\mathrm{C}$ bonds (bridge configuration). Again, as is the case of one-Si fullerene, bridge and edge configurations are topologically very similar. Figure 4 shows the lowest energy substitutional, on-top, and bridge isomers of $\mathrm{C}_{n} \mathrm{Si}_{2}$ heterofullerenes, obtained with the Tersoff potential.

As discussed above, the competition between exohedral and substitutional structures in one-Si heterofullerene is controlled by the tendency to form fullerene cages with an even number of atoms. This is accomplished in the even and odd heterofullerenes by adopting substitutional and exohedral configurations, respectively. However, for two-Si heterofullerenes this competition becomes a "true" question since, for even heterofullerenes, both isomers have an even fullerene as the undoped underlying structure. To address this problem we have fully optimized some selected substitutional, edge, and bridge structures of $\mathrm{C}_{58} \mathrm{Si}_{2}$ and $\mathrm{C}_{60} \mathrm{Si}_{2}$, using the DFT formalism. Previous DFT studies ${ }^{11,15,16}$ concentrate on substitutional $\mathrm{C}_{58} \mathrm{Si}_{2}$. A different lowest energy substitutional isomer was obtained depending on the details of the calculation. In Fig. 5 we present the lowest energy substitutional and exohedral isomers of $\mathrm{C}_{58} \mathrm{Si}_{2}$ and $\mathrm{C}_{60} \mathrm{Si}_{2}$ obtained with DFT. The minimum energy structure of $\mathrm{C}_{58} \mathrm{Si}_{2}$ is the substitutional isomer whereas the exohedral-edge configuration is the preferred structure of $\mathrm{C}_{60} \mathrm{Si}_{2}$. It is interesting to notice that the undoped underlying structure of the ground state of $\mathrm{C}_{58} \mathrm{Si}_{2}$ and $\mathrm{C}_{60} \mathrm{Si}_{2}$ is, in both cases, $\mathrm{C}_{60}$. Clearly, the

lowest energy structures of these two heterofullerenes are a consequence of the special stability of $\mathrm{C}_{60}$ with respect to the neighboring fullerenes.

The lowest energy substitutional isomer of $\mathrm{C}_{58} \mathrm{Si}_{2}$ has the two Si atoms in a "para" configuration of a hexagon (the $\mathrm{Si}$ atoms are in two opposite vertices of the hexagon). It is remarkable that the Tersoff potential produces the same lowest energy substitutional isomer. Moreover, the family of substitutional isomers, as calculated with the Tersoff potential, spans an energy interval of about $1 \mathrm{eV}$, in perfect agreement with the DFT result. ${ }^{15}$ At "low" Si concentrations (for $\mathrm{C}_{58} \mathrm{Si}_{2}$ and $\mathrm{C}_{60} \mathrm{Si}_{2}$ ), the two substitutional $\mathrm{Si}$ atoms are not nearest neighbors since the formation of a $\mathrm{Si}-\mathrm{Si}$ bond does not compensate for the extra local distortion produced in the cage, due to the larger $\mathrm{Si}-\mathrm{Si}$ bond length. However, on the basis of ab initio calculations, ${ }^{16}$ grouping of the $\mathrm{Si}$ atoms is expected for higher concentrations. On the other hand, the two $\mathrm{Si}$ atoms group together on the fullerene surface of the exohedral isomers. Clearly, a $\mathrm{Si}-\mathrm{Si}$ bond can be accommodated in the surface without stressing the fullerene cage. Photofragmentation experiments ${ }^{13}$ suggest clustering of the $\mathrm{Si}$ atoms on the fullerene surface, also for higher Si concentrations.

Clearly, it is not an easy task for an empirical potential to produce the correct minimum energy structure and isomer hierarchy of small clusters as a function of cluster size and composition. Despite its limitations, we have shown that the Tersoff potential retains the most important structural and energetic characteristics of Si-doped fullerenes, what makes it a very useful tool for investigating the dynamical and thermal characteristics of those systems. The rest of this section is devoted to that study. 


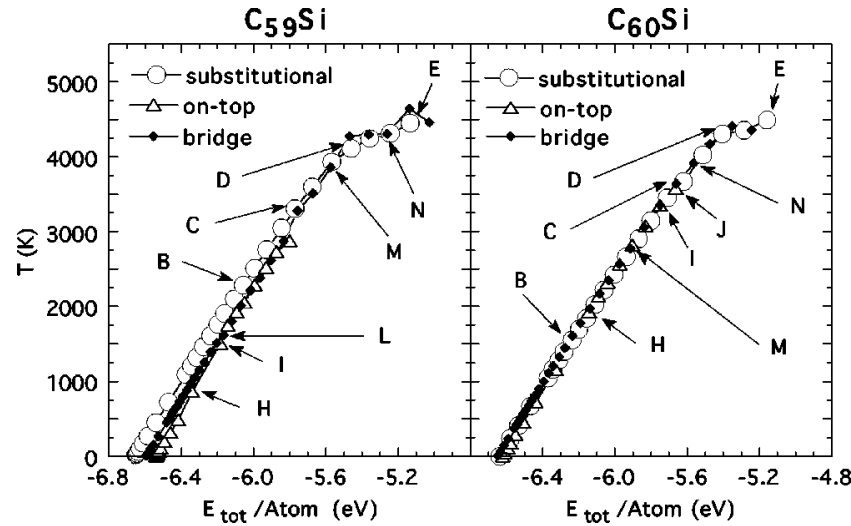

FIG. 6. Caloric curves of $\mathrm{C}_{59} \mathrm{Si}$ and $\mathrm{C}_{60} \mathrm{Si}$. The different curves in each panel correspond to different branches of the caloric curve generated from the isomers shown in Fig. 2: substitutional (circles), on-top (triangles), and bridge (solid diamonds) isomers. The arrows, labeled as $\mathrm{B}-\mathrm{E}, \mathrm{H}-\mathrm{I}$, and $\mathrm{L}-\mathrm{N}$ for $\mathrm{C}_{59} \mathrm{Si}$ and $\mathrm{B}-\mathrm{E}, \mathrm{H}-\mathrm{J}$, and $\mathrm{M}-\mathrm{N}$ for $\mathrm{C}_{60} \mathrm{Si}$, indicate the onset of different structural transformations along the trajectories originating in the substitutional, the on-top, and the bridge isomers, respectively. Corresponding snapshots are shown in Figs. 8 and 9 for $\mathrm{C}_{59} \mathrm{Si}$ and $\mathrm{C}_{60} \mathrm{Si}$, respectively (see text for details).

\section{Thermal behavior of one-Si heterofullerene}

Extensive constant energy molecular dynamics (MD) simulations have been performed as a function of temperature, from $T=0 \mathrm{~K}$ until the thermal decomposition (evaporation of atoms or fragmentation) of the heterofullerenes occurs $(T \sim 4000-5000 \mathrm{~K})$. The average thermal and structural characteristics of the heterofullerenes as a function of temperature are embodied in the caloric curves (temperature vs total energy) and in the $\delta$ function $^{31}$ [given in Eq. (8)], respectively. Different branches of the caloric curves are generated by heating up different isomers. The heating of the heterofullerenes is accomplished through the scaling of the velocities of all atoms, between successive constant energy MD runs. The (kinetic) temperature, $T$, is defined in the usual way in dynamical simulations,

$$
k_{B} T=\frac{2}{3 n-6}\left\langle E_{\mathrm{kin}}\right\rangle_{t},
$$

where \langle\rangle$_{t}$ represents the time average over the entire trajectory, $E_{\text {kin }}$ is the instantaneous kinetic energy of the heterofullerene, $n$ is the number of atoms, and $k_{B}$ is the Boltzmann constant. The $\delta$ function (relative root mean square bond length fluctuations) is given by

$$
\delta=\frac{2}{n(n-1)} \sum_{i<j} \frac{\left(\left\langle r_{i j}^{2}\right\rangle_{t}-\left\langle r_{i j}\right\rangle_{t}^{2}\right)^{1 / 2}}{\left\langle r_{i j}\right\rangle_{t}},
$$

where $r_{i j}$ is the instantaneous distance between atoms $i$ and $j$ and the sum runs over all the pairs of atoms in the heterofullerene. For heteroatomic systems it is interesting to consider partial $\delta \mathrm{s}$ ( $\delta_{\alpha \alpha}$ and $\delta_{\alpha \beta}$, where $\alpha$ and $\beta$ stand for the atom type) calculated by restricting the summations in Eq. (8) to pairs of like or unlike atoms, respectively. Differences among the partial $\delta$ s indicate different mobilities of the two types of atoms.

Figures 6 and 7 show the caloric and the $\delta$ curves, respectively, generated from the lowest energy substitutional,

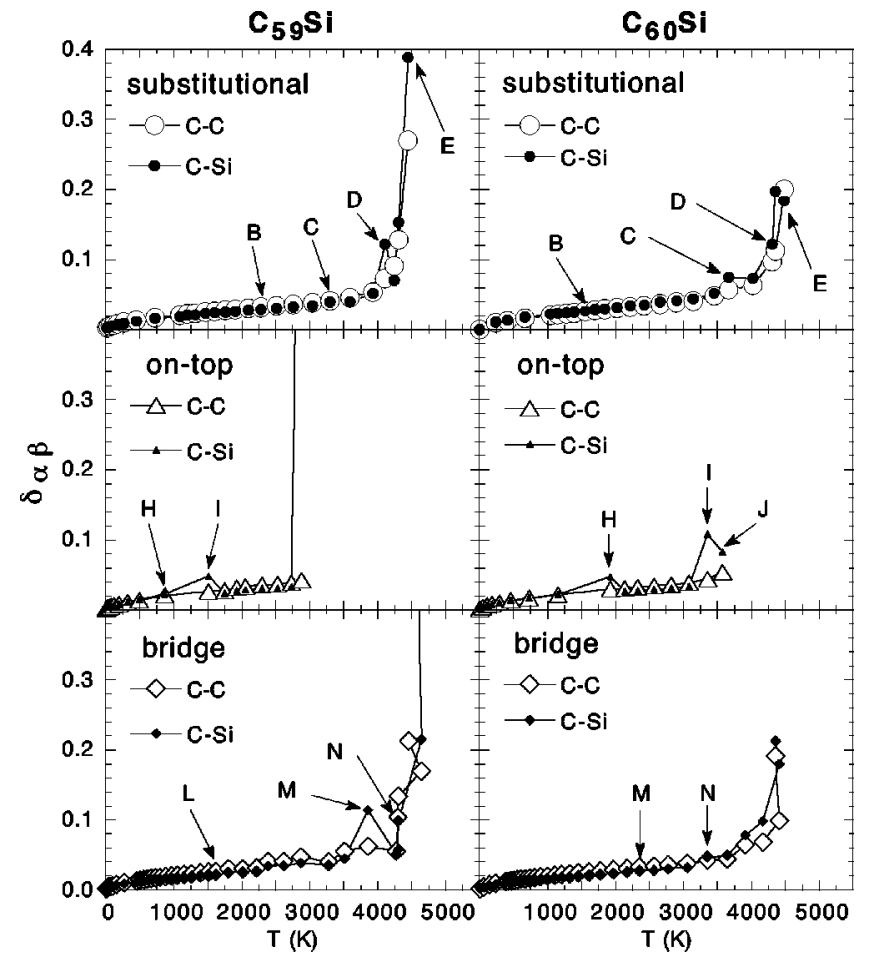

FIG. 7. Partial $\delta \mathrm{s}$ of $\mathrm{C}_{59} \mathrm{Si}$ and $\mathrm{C}_{60} \mathrm{Si}$ as a function of temperature for the same isomers considered in Fig. 6 . The arrows have the same meaning as in that figure. $\delta_{\mathrm{CC}}$ : open symbols, and $\delta_{\mathrm{CSi}}$ : full symbols.

exohedral-on-top, and exohedral-bridge isomers of $\mathrm{C}_{59} \mathrm{Si}$ and $\mathrm{C}_{60} \mathrm{Si}$ (shown in Fig. 2). The corresponding curves for $\mathrm{C}_{61} \mathrm{Si}$ and $\mathrm{C}_{58} \mathrm{Si}$ exhibit, respectively, similar behavior. The small horizontal separation between the different branches of the caloric curves at zero temperature reflects the small energy difference between the corresponding isomers. Although these energy differences are of the order of the thermal energy at room temperature, much higher temperatures are required to observe structural transitions between those isomers, within the time window of our simulations (see below). At low temperatures $(T<1000 \mathrm{~K})$ the caloric curves are linear functions, as expected from a classical treatment, and, the small $(<10 \%)$ values of $\delta$ reflect solidlike structures $^{31}$ in which the atoms perform harmonic oscillations about their equilibrium positions. With increasing temperature $(T \sim 1000-4000 \mathrm{~K})$ the anharmonic contributions start to be significant and the slopes of the caloric curves begin to decrease slowly. At these intermediate temperatures the heterofullerenes may experience occasional (nondiffusive) isomerization transitions. Therefore, these structural changes have only a minor reflection in the caloric curve (as small jumps or increase in the slope) and the $\delta$ values remain small. The only noticeable feature are some small peaks exhibited by $\delta_{\mathrm{CSi}}$, without a related feature in $\delta_{\mathrm{CC}}$, which correspond to structural transformations involving displacements of the $\mathrm{Si}$ atom on the fullerene structure. At $T$ $\sim 4000 \mathrm{~K}$ the caloric curves level off and the $\delta$ s exhibit a sharp increase, ${ }^{31}$ indicating the beginning of the solid- to liquidlike transition. However, similarly to the case of pure carbon fullerenes, ${ }^{22,30}$ thermal decomposition of the heterofullerenes occurs before reaching a liquidlike regime which 


\section{Substitutional}

A

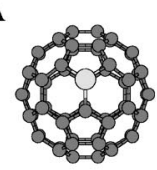

$0 \mathrm{~K}$

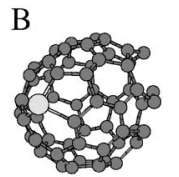

$2300 \mathrm{~K}$

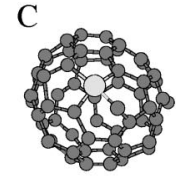

$3300 \mathrm{~K}$

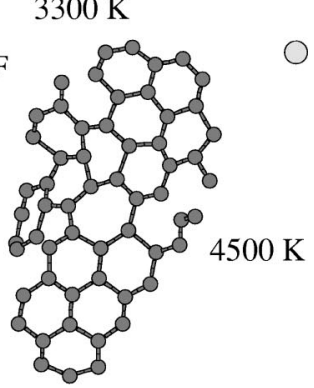

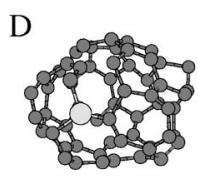

$4100 \mathrm{~K}$

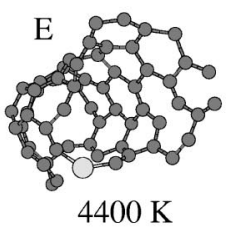

On-top

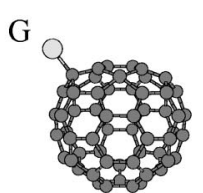

$0 \mathrm{~K}$

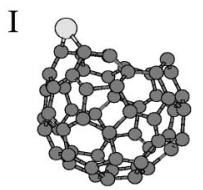

$1500 \mathrm{~K}$

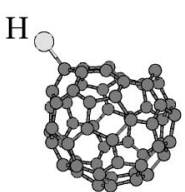

$900 \mathrm{~K}$

$\mathrm{J}$

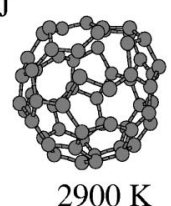

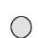

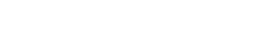




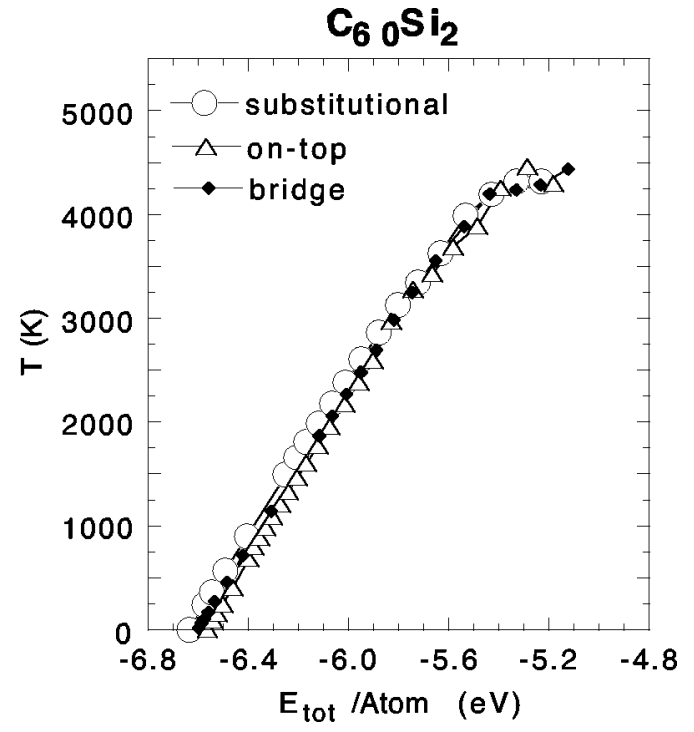

FIG. 10. Caloric curve of $\mathrm{C}_{60} \mathrm{Si}_{2}$. The different curves correspond to different branches of the caloric curve generated from the isomers shown in Fig. 4: substitutional (circles), attached (triangles), and bridging (solid diamonds) isomers.

The first structural transformation observed consists in the opening of windows $(\mathrm{OW})$ in the fullerene surface through the break up of either $\mathrm{C}-\mathrm{Si}$ or $\mathrm{C}-\mathrm{C}$ bonds (see Fig. $8 \mathrm{~B}, \mathrm{H}$, L and Fig. 9 B, M). Due to the local character of this transformation no special features are observed neither in the caloric nor the $\delta$ curves. Notice that this transformation occurs at lower temperatures $(T<1000 \mathrm{~K})$ in the on-top isomers of even heterofullerenes than in the other isomers ( $T$ $>1500 \mathrm{~K}$ ). This correlates with the lower stability of the former isomers. At about $1500 \mathrm{~K}$ the on-top isomers transform into bridge isomers by inserting the $\mathrm{Si}$ atom in the fullerene cage (see Fig. $8 \mathrm{I}$ and Fig. $9 \mathrm{H}$ ), and at higher temperatures $(T \sim 2500-3500 \mathrm{~K})$ the bridge isomers transform into substitutional isomers (see Fig. $8 \mathrm{M}$ and Fig. $9 \mathrm{~N}$ ). The successive transformation of the on-top isomer into bridge and then into a substitutional structure is observed for $\mathrm{C}_{60} \mathrm{Si}$ (see Fig. $9 \mathrm{~J}$ ). These isomerization transitions produce a small peak in $\delta_{\mathrm{CSi}}$ but no special feature is observed in $\delta_{\mathrm{CC}}$ since they are mainly produced by displacements of the $\mathrm{Si}$ atom. Our dynamical simulations demonstrate the transformation of "energized" exohedral heterofullerenes into substitutional heterofullerenes. This transformation has been actually observed upon laser irradiation of samples of exohedral heterofullerenes. ${ }^{12}$ Above $3000 \mathrm{~K}$, a high mobility of the $\mathrm{Si}$ atom on the fullerene surface is observed, accompanied by frequent changes in its coordination, whereas the underlying fullerene remains quite intact (see Fig. 8 C, D, N and Fig. $9 \mathrm{C}, \mathrm{D}, \mathrm{I}) . \delta_{\mathrm{CSi}}$ (Fig. 7) exhibits small features which reflect the high mobility of the silicon atom. At higher temperatures $(T \sim 4000-4500 \mathrm{~K})$ the solid to liquidlike transition begins. Substitutional isomers and the bridge isomers of even heterofullerenes experience, similarly to the case of all carbon fullerenes, strong structural transformations destroying the cage structure and forming tubular (see Fig. 8 E, O and Fig. 9 E, F) or graphiticlike planar structures (see Fig. $8 \mathrm{~F}$ ). However, the evaporation of the $\mathrm{Si}$ atom (and in fewer

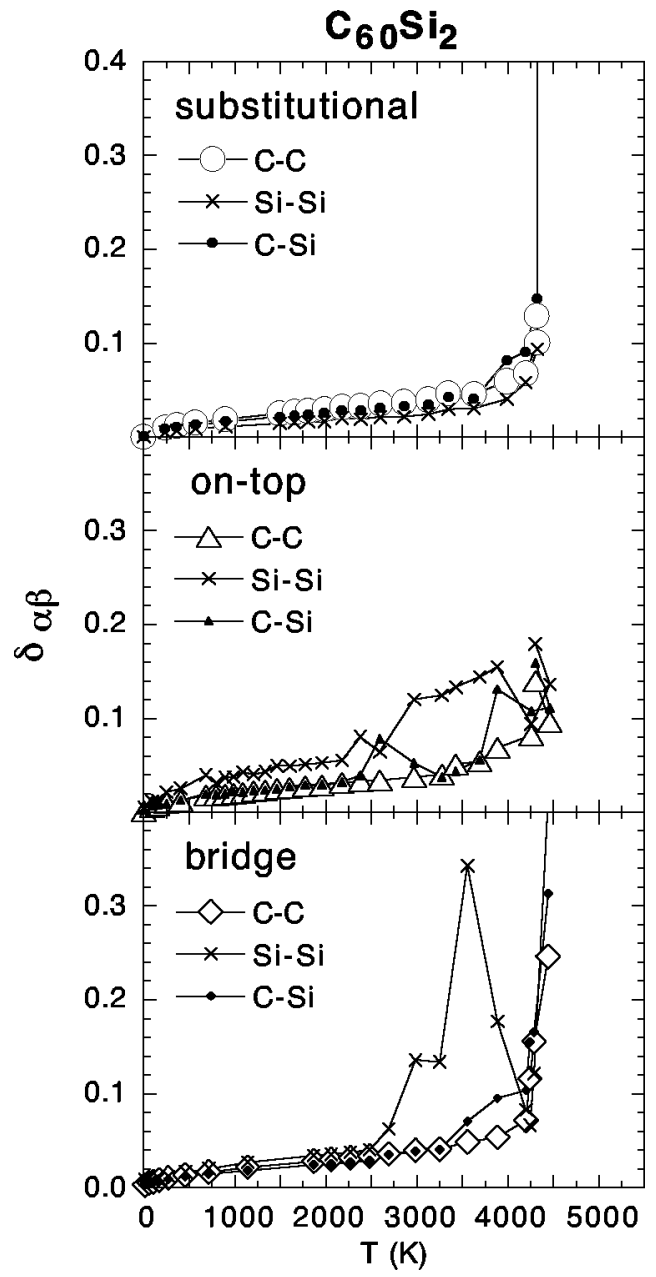

FIG. 11. Partial $\delta s$ of $\mathrm{C}_{60} \mathrm{Si}_{2}$ as a function of temperature for the same isomers considered in Fig. $10 . \delta_{\mathrm{CC}}$ : open symbols, $\delta_{\mathrm{SiSi}}$ : crosses, and $\delta_{\mathrm{CSi}}$ : full symbols.

cases $\mathrm{C}$ or $\mathrm{C}_{2}$ units) starts before the fullerenes have reached the liquidlike phase. On the other hand, on-top isomers and the bridge isomers of odd heterofullerenes sublimate (evaporate atoms) directly from the fullerene structure (see Fig. $8 \mathrm{~J}$ and Fig. $9 \mathrm{~K}, \mathrm{O}$ ). Sublimation from almost undistorted fullerene structures correlates with the low stability of on-top silicon and with the tendency to form even fullerenes.

\section{Thermal behavior of two-Si heterofullerenes}

We have also investigated the thermal stability of two-Si heterofullerenes containing 58,60, and 78 carbon atoms. As a representative case, Figs. 10 and 11 present the caloric and the $\delta$-curves, respectively, generated from the isomers of $\mathrm{C}_{60} \mathrm{Si}_{2}$ shown in Fig. 4. The presence of a second silicon atom does not introduce drastic changes in the main thermal and structural characteristics of the heterofullerenes. It is apparent, however, a higher mobility of the exohedral (on-top and bridge) silicon atoms at intermediate temperatures $(T$ $>2500 \mathrm{~K})$. This higher mobility is reflected by the increase of $\delta_{\mathrm{SiSi}}$ (see Fig. 11). It is interesting to notice that, similarly to the case of one-Si fullerenes, the exohedral on-top and bridge isomers transform into substitutional isomers at intermediate temperatures $(T \sim 2000-3000 \mathrm{~K})$. Our results indi- 
cate that this structural transformation, taking $\mathrm{Si}$ atoms from exohedral into substitutional configurations, could be repeated several times to obtain Si-rich substitutional fullerenes. Recent experiments ${ }^{14}$ indicate that at least seven $\mathrm{Si}$ atoms can be substituted in $\mathrm{C}_{60}$. At high temperatures $(T \geqslant 4000 \mathrm{~K})$, on-top isomers sublimate from almost undistorted cage structures whereas the substitutional and the bridge isomers transform into more open tubular or planar structures before the evaporation of atoms begins.

\section{SUMMARY}

We have performed extensive Molecular Dynamics simulations to investigate the structural and thermal features of Si-doped fullerenes as a function of fullerene size and $\mathrm{Si}$ concentration (for one and two $\mathrm{Si}$ atoms). Both, the manybody Tersoff potential and $a b$ initio DFT techniques have been used to describe the interatomic interactions in the heterofullerenes. First, we focused on the competition between exohedral and substitutional doping. The minimum energy structures of the heterofullerenes are based on fullerenes with an even number of atoms. The dopant $\mathrm{Si}$ atoms may be, either, substitutionally incorporated in the fullerene structure, or exohedrally attached to the fullerene surface. Thus, the substitutional isomer is the minimum energy structure of even heterofullerenes whereas the exohedral isomer is the lowest energy structure of odd heterofullerenes. These results are in perfect agreement with mobility experiments. ${ }^{10}$ Exohedral attachment of $\mathrm{Si}$ in preformed fullerenes, is an exothermic reaction whereas the substitution of $\mathrm{C}$ by $\mathrm{Si}$ in even fullerenes is an endothermic process. As a consequence, the soft landing of $\mathrm{Si}$ atoms on the surface of preformed fullerenes results in the formation of exohedral heterofullerenes. These results explain the experimental production of exohedral heterofullerenes through the mixing of $\mathrm{C}_{60}$ fullerenes and $\mathrm{Si}$ vapors. ${ }^{12}$ At the first stages of multiple doping (two $\mathrm{Si}$ atoms), the two $\mathrm{Si}$ atoms are not nearest neighbors in the substitutional isomers; however, they group together on the surface of the exohedral isomers.

A variety of structural transformations of Si-doped heterofullerenes have been identified and characterized as a function of temperature. Those transformations include: (i) local structural changes without diffusion of atoms (opening of windows in the cage), (ii) isomerization transitions between exohedral and substitutional isomers, (iii) drastic structural changes involving first diffusion of the silicon atom(s) and then diffusion of all the atoms in the heterofullerene, (iv) eventual destruction of the fullerene cage structure, and, finally, (v) sublimation of atoms (which happens before the liquidlike phase is reached). The conversion of exohedral into substitutional isomers has been observed upon laser irradiation of samples of exohedral heterofullerenes. $^{12}$

Interestingly, silicon doping does not destroy the structural and thermal stability of the fullerenes while changing their electronic properties. This could be used to develop new assembled materials based on Si-doped fullerenes with novel structural and electronic properties as compared to other $\mathrm{C} / \mathrm{Si}$ systems.

\section{ACKNOWLEDGMENTS}

Work supported by the DGES of Spain (Grant Nos. PB98-0345 and MAT2002-04499-C02), European Community (RTN-COMELCAN), and Junta de Castilla y León (Grant Nos. VA28/99 and CO01/102). M.J.L. acknowledges support from the Spanish MCYT under the "Ramón y Cajal" Program.

${ }^{1}$ H. W. Kroto, J. R. Heath, S. C. O'Brien, R. F. Curl, and R. E. Smalley, Nature (London) 318, 162 (1985).

${ }^{2}$ W. Krätschmer, L. D. Lamb, K. Fostiropoulos, and D. R. Huffman, Nature (London) 347, 354 (1990).

${ }^{3}$ J. R. Heath, S. C. O'Brien, Q. Zhang, Y. Liu, R. F. Curl, H. W. Kroto, F. K. Tittel, and R. E. Smalley, J. Am. Chem. Soc. 107, 7779 (1985).

${ }^{4}$ See, for example, P. Weis, R. D. Beck, G. Bräuchle, and M. M. Kappes, J. Chem. Phys. 100, 5684 (1994).

${ }^{5}$ See, for example, A. R. Kortan, N. Kopylov, E. Ozdas, A. P. Ramírez, R. M. Fleming, and R. C. Haddon, Chem. Phys. Lett. 223, 501 (1994).

${ }^{6}$ T. Guo, C. Jin, and R. E. Smalley, J. Phys. Chem. 95, 4948 (1991).

${ }^{7}$ H. J. Muhr, R. Nesper, B. Schnyder, and R. Kîtz, Chem. Phys. Lett. 249, 399 (1996).

${ }^{8}$ J. C. Hummelen, B. Knight, J. Pavlovich, R. González, and F. Wudl, Science 269, 1555 (1995).

${ }^{9}$ T. Kimura, T. Sugai, and H. Shinohara, Chem. Phys. Lett. 256, 269 (1996).

${ }^{10}$ J. L. Fye and M. F. Jarrold, J. Phys. Chem. A 101, 1836 (1997).

${ }^{11}$ C. Ray, M. Pellarin, J. Lermé, J. L. Vialle, M. Broyer, X. Blase, P. Mélinon, P. Kéghélian, and A. Perez, Phys. Rev. Lett. 80, 5365 (1998).

${ }^{12}$ I. M. L. Billas, F. Tast, W. Branz, N. Malinowski, M. Heinebrodt, T. P. Martin, M. Boero, C. Massobrio, and M. Parrinello, Eur. Phys. J. D 9, 337 (1999).

${ }^{13}$ M. Pellarin, C. Ray, J. Lermé, J. L. Vialle, M. Broyer, and P. Mélinon, J. Chem. Phys. 112, 8436 (2000).

${ }^{14}$ M. Pellarin, C. Ray, J. Lermé, J. L. Vialle, M. Broyer, X. Blase, P. Kéghélian, P. Mélinon, and A. Perez, J. Chem. Phys. 110, 6927 (1999).

${ }^{15}$ I. M. L. Billas, C. Massobrio, M. Boero, M. Parrinello, W. Branz, F. Tast, N. Malinowski, M. Heinebrodt, and T. P. Martin, J. Chem. Phys. 111, 6787 (1999).

${ }^{16}$ C.-C. Fu, M. Weissmann, M. Machado, and P. Ordejón, Phys. Rev. B 63, 085411 (2001).

${ }^{17}$ E. Kolodney, B. Tsipinyuk, and A. Budrevich, J. Chem. Phys. 100, 8542 (1994).

${ }^{18}$ S. D. Leifer, D. G. Goodwin, M. S. Anderson, and J. R. Anderson, Phys. Rev. B 51, 9973 (1995); M. R. Stetzer, P. A. Heiney, J. E. Fischer, and A. R. McGhie, ibid. 55, 127 (1997).

${ }^{19}$ J. Tersoff, Phys. Rev. B 37, 6991 (1988); Phys. Rev. Lett. 61, 2879 (1988).

${ }^{20}$ J. Tersoff, Phys. Rev. B 39, 5566 (1989).

${ }^{21}$ G. C. Abell, Phys. Rev. B 31, 6184 (1985).

${ }^{22}$ M. J. López, P. A. Marcos, A. Rubio, and J. A. Alonso, Z. Phys. D: At., Mol. Clusters 40, 385 (1997).

${ }^{23}$ L. Verlet, Phys. Rev. 159, 98 (1967); W. C. Swope and H. C. Andersen, J. Chem. Phys. 76, 637 (1982).

${ }^{24}$ See, e.g., M. B. Nardelli, C. Brebec, A. Maiti, C. Roland, and J. Bernholc, Phys. Rev. Lett. 80, 313 (1998); M. J. López, A. Rubio, J. A. Alonso, S. Lefrant, K. Méténier, and S. Bonnamy, ibid. 89, 255501 (2002).

${ }^{25}$ The DACAPO code can be found in the Web page www.fysik.dtu.dk/ CAMPOS/

${ }^{26}$ D. H. Vanderbilt, Phys. Rev. B 41, 7892 (1990).

${ }^{27}$ J. P. Perdew et al., Phys. Rev. B 46, 6671 (1992).

${ }^{28}$ J. Lu, Y. Zhou, S. Zhang, X. Zhang, and X. Zhao, Chem. Phys. Lett. 343, 39 (2001).

${ }^{29}$ P. W. Fowler and D. E. Manolopoulos, in An Atlas of Fullerenes (Clarendon, Oxford, 1995).

${ }^{30}$ P. A. Marcos, J. A. Alonso, A. Rubio, and M. J. López, Eur. Phys. J. D 6, 221 (1999).

${ }^{31}$ F. A. Lindemann, Z. Phys. 11, 609 (1910). 